\title{
DISCUSSION.
}

Mr. John G. A. Rhodin : I have read this Paper with great interest, and the subject is one of great fascination to me. I must, however, confess that, although I have tried to follow the resuscitation of the electron theory since the discovery of the Zeeman effect, my mind fails to understand what the actual gain has been to our science with regard to the interpretation of the more ordinary phenomena. Like most chemical theories, it seems to me to be a reversion to the ancient method of the quadrature of closed figures. The same applies to the atomic theory. To my mind, the atomic weights are nothing else but numerical solutions of differential coefficients, relating to acting masses and gravitation during a chemical reaction. Whether they are circular or elliptical functions, as has been sometimes suggested, is not known. To make matters simple, Dalton conceived a quadrature with the value for hydrogen as unity. This is in itself a permissible operation, but I doubt whether any scientific man is convinced about the indivisibility of the atoms, except as an image to elucidate the constancy of a ratio between two masses. Infinite divisibility is the necessary supposition for the proof of most problems in the calculus, if our reason is going to be satisfied. Similarly, this must be the case with matter in general. Take a solution of $\mathrm{HCl}$. We can easily conceive that the

* See Kohlrausch and Grüneisen, Sitzungsber. Berlin Akad., 40, July 28, 1904. 


\section{THE ELECTRON THEORY TO ELECTROLYSIS}

centres of motion in the form of $\mathrm{HCl}$ molecules may differ as to mass, as long as $\mathrm{H}: \mathrm{Cl}=$ constant. In other words, the atomic weights do not exist, except where a reaction takes place. The greatest supporter of Dalton's theory -J. J. Berzelius-must have had a similar idea, as he doggedly stuck to the term equivalent, which no doubt illustrates the only actual knowledge which we possess on this subject. In the same manner all chemists in actual practice cease to bother about atoms very soon after leaving the university, simply retaining the well-established numerical ratios as a guide. If we now turn to what I should like to call "metaphysical chemistry," or "metachemical physics," we shall find that our knowledge is confined to experimentally determined numbers and mathematical relationships. The explanatory hypotheses are simply more or less crude images, generally encouraged by the simplicity of numerical calculations in limiting cases. The well-known rule, $\lim a^{2}=2 a$, may thus be said to be the basis of the electrolytic dissociation theory. Hence the eternal search for constants for infinite dilution. This has, however, become a fashion.

With regard to electrical and electromagnetic phenomena, it was necessary to suppose a medium unaffected by gravitation, or one very slightly so, but capable of immense velocity of motion. In this way we got the ether and the electrons. Both are simple conceptions to explain action at a distance. Newton's light corpuscles, which emanated from a luminous body, were the prototype of the electrons. If some theory of harmonic whirls could have been easily elaborated to explain interference and polarisation phenomena, we might not have had the ether. Transverse vibrations in a kind of solid ether were more easily treated mathematically. Hence, exit emanation, enter undulation and ether. The genius of men like Gauss, Weber, Maxwell, Helmholtz, and others, gave the ether a long lease of life, which I presume has not run out as yet. Certain other phenomena, studied in the sixties, and a short decennium ago the Zeeman effect, could be explained by corpuscles of immense mobility, hence enter electrons !

Now, with regard to electrolysis, I contend that most phenomena are very easily treated by the fairly simple theory of ions as a fundamental principle. What we require is a tabulated knowledge of phenomena in concentrated solutions and with finite current densities. The nature of the charges carried is of so much less consequence, as we are never likely to have a look at them. Why make experiments to support a favourite notion which can never be proved to the satisfaction of our senses? Even the ions are somewhat too excessive a dose of credulity to be medicinal. Why fill them with a similar conception?

The answer is near at hand. To discover new, valuable scientific facts and laws is rather difficult, hence unconsidered trifles have to be magnified. Even the realm of ghosts has been invaded by scientific men in all earnest. Theories are evolved on the most slender basis of facts, and just now they are so many that I am willing to issue a challenge that no man in the world can define which are the accepted ones. That the great liking for the outre which characterises our time has something to with this is certain, as some of the most well-known facts are left without an explanation. How do you, for instance, reconcile the vapour density and valence of $\mathrm{FeCl}_{3}$ with the theory of indivisible atoms? Why does hard-drawn pure copper wire gain only 2 per cent. in conductivity by annealing, when hard-drawn silver gains 4 per cent. (approximately)? Why does an annealed, soft, pure copper wire lose density by hard drawing ? Why do we reckon copper as not being a hydrogen-displacing metal when, as a matter of fact, copper dissolves fairly rapidly in $10 \mathrm{~N} \mathrm{HCl}$ at $80^{\circ} \mathrm{C}$. with evolution of hydrogen? I have personally 
verified this with pure electrolytic copper, and Mr. Bertram Blount, who doubted it, did the same to his own satisfaction. That volumes could be filled with such queries is evident to most chemists who do not take chemistry as the Athanasian Creed.

In conclusion, what $I$ mean is this. We do not at present want any more theories-we want a generalisation of existing theories of such a nature that one notion does not contradict another. The rest would not suffer by being noted as observed facts. An international committee for this purpose would probably do more good than a certain other one, which compounded a terminology of organic chemistry which nobody knows or accepts. We practical men have a certain claim that our science should be so systematised as to be available to the average intelligence without unduly taxing one's memory, which after all has to be used for strictly utilitarian purposes.

Dr. H. Borns had no doubt that the author could have given them a great deal more if time had permitted, but he regretted that the author had not found it possible to indicate the lines on which he conceived metallic conduction by electrons. He did not understand in which sense he was to regard the electron theory as an extension of the ionic theory of electrochemistry. In its modern conception the electron theory might be dated from J. J. Thomson's deduction of 1887 , that an electrically charged body in motion should behave as if its mass were increased by a certain quantity. He could not quite follow the argument concerning mobility of electrons in copper and in hydrochloric acid, and why there should be electrons in a vacuum and in a metal, but not in a liquid. What became of the electrons when a metal was fused? Perhaps the author would also explain the condensation taking place on a charged drop a little further. The author had referred to Drude, Schuster, and J. J. Thomson. He might have added the names of Kaufmann, Riecke, Lorentz and Abraham, and others; to the latter two we owed complete theories. The author had not referred to the difficulties of the electron theory, to the Hall effect, nor to the fact that metallic conduction seemed to become badly involved when we assumed positive in addition to negative electrons. Had not Kaufmann's recent experiments, as discussed by the Naturforscher-Versammlung at Stuttgart, left the two theories mentioned rather under a cloud, moreover?

Dr. T. M. Lowry thanked the author for his fascinating and suggestive Paper. The numerical data given in reference to the number, size, and velocity of the electrons were remarkably definite, and he would be glad if the author could refer him to the original papers in which these figures had been deduced.

Mr. Fournier had stated that electrolytes had positive temperature coefficients. This was by no means universally true. In many cases the conductivity reached a maximum, and at higher temperatures the coefficient was negative. The question had been fully discussed in a recent paper (Bousfield and Lowry, Proc. Roy. Soc., 1902, 71. 42).

The author would perhaps be interested to know that quantitative determinations of the hydrations of the ions were being made by a number of investigators, including Biltz, H. C. Jones, and Bousfield. The latter had arrived at the conclusion that the potassium and chlorine ions were combined with about 5 molecules of water, but the more sluggish lithium ion with about 20 molecules. The bearing of ionic hydration on the theory of electrolysis had already been discussed before the Society (Trans. July, rgo5).

$\mathrm{He}$ would like to know the authority for the statement that "when an ion contains 50 or 60 atoms it appears to have a minimum mobility which is not 
perceptibly diminished by a further addition of atoms." The only law of which he was aware was in reference to the temperature coefficients of mobility, which in the case of the more massive ions reached a maximum limit identical with the temperature coefficient of the fluidity of water.

Mr. N. T. M. Wilsmore (partly communicated) : I must beg to differ from some of the previous speakers, as I consider, with Mr. Fournier-d'Albe, that the electron theory helps to simplify our views of the mechanism of electrolytic phenomena. This is more especially the case on account of the fact that so far only " negative" electrons appear to be capable of existence in the free state, and that, therefore, most electrochemical reactions may be looked upon as being, to a great extent, merely interchanges of these negative electrons.

If it could be proved that "positive" electrons were incapable of existence, the name "electron" might be used as an abbreviation of "negative electron"; but, as long as that proof is wanting, one might, perhaps, be allowed to condense the latter expression into "negatron." *

On the electron theory of J. J. Thomson, Drude, \&c., the electrolytic potentials of the elements are believed to be due to what may be called the dissociation pressures of the free electrons-according to J. J. Thomson, negative electrons-which they contain. This hypothesis is supported by the observation of J. J. Thomson $\dagger$ that the strongly "electro-positive" elements spontaneously give off negatrons, and by the results obtained by Ramsay and Spencer $\ddagger$ from a study of the action of ultra-violet light on various elements, the rates at which these give off negatrons being found proportional to their positions in the electrochemical series.

It is not necessary to assume that the interchange of electrons at an electrode is always between atoms of the same kind. For instance, the action on a "reduction" electrode, with, say, a ferrous salt as electrolyte, is most simply represented thus-

$$
\mathrm{Fe} \cdot-\mathrm{\theta}=\mathrm{Fe}^{\cdots}
$$

a ferrous ion becoming a ferric by giving up a negatron to the (platinum) clectrode. Similarly the reaction on a typical "oxidation" electrode may be shown thus-

$$
\mathrm{MnO}_{4}{ }^{\prime}+8 \mathrm{H} \cdot+5 \mathrm{\theta}=\mathrm{Mn} \cdot+{ }_{4} \mathrm{H}_{2} \mathrm{O}
$$

the electrolyte, permanganate + dilute acid, taking five negatrons from the (platinum) electrode, with formation of manganous salt and water. The direct reaction in solution between permanganate and ferrous iron would be-

$$
\mathrm{MnO}_{4}{ }^{\prime}+8 \mathrm{H} \cdot+5 \mathrm{Fe}^{\cdots}=\mathrm{Mn} \cdot+{ }_{4} \mathrm{H}_{2} \mathrm{O}+{ }_{5} \mathrm{Fe}^{\cdots}
$$

the corresponding interchange of negatrons being here between the ferrous ions and the hydrions. Or ferrous iron may be supposed to react with nascent oxygen in acid solution as follows-

$$
\begin{aligned}
2 \mathrm{Fe} \cdot+\mathrm{H}_{2} \mathrm{O}+\mathrm{O} & =2 \mathrm{Fe} \cdots+2 \mathrm{OH}^{\prime} \\
2 \mathrm{OH}^{\prime}+2 \mathrm{H} \cdot & =2 \mathrm{H}_{2} \mathrm{O}
\end{aligned}
$$

* This word has little claim to acceptance on æsthetic or etymological grounds : but electrical science appears to be saddled permanently with the terms "positive" and "negative," although their connotation has lately suffered reversal, "positive" now meaning " excess of matter" instead of "excess of electricity."

$\dagger$ Phil. Mag. [6] x 584 (1905). $\quad$ Ibid. xii. 397 (1906). 
hydroxidions being the carriers of the negatrons between the ferrous ions and the hydrions. The net reaction is of course-

$$
2 \mathrm{Fe}^{\cdots}+2 \mathrm{H} \cdot+\mathrm{O}=2 \mathrm{Fe}^{\cdots}+\mathrm{H}_{2} \mathrm{O} \text {. }
$$

Electrode reactions of complex ions may also be viewed in this way. For example, according to Bodländer and Eberlein * a solution of silver in potassium cyanide contains the silver in the form of a complex anion $\mathrm{Ag}(\mathrm{CN})_{3}{ }^{\prime \prime}$. According to the views till lately in fashion the reactions of this complex at an electrode would take place in two stages-

$$
\begin{aligned}
& \mathrm{Ag}(\mathrm{CN})_{3}{ }^{\prime} \rightleftarrows \mathrm{Ag} \cdot+3 \mathrm{CN}^{\prime} \\
& \mathrm{Ag} \cdot+\mathrm{O} \rightleftarrows \mathrm{Ag} \text { (metal), }
\end{aligned}
$$

viz. (taking the reactions from left to right), first a dissociation of the complex, giving $\mathrm{Ag} \cdot$ ion, followed by the discharge and deposition of this ion by a negatron from the cathode. Haber thas lately shown, however, that the probable concentration of the $\mathrm{Ag} \cdot$ ions (about ro $^{-24}$ ) is far too small to allow of their being the carriers of an appreciable current into or out of the solution. The reactions at an electrode must therefore be represented thus-

$$
\mathrm{Ag}(\mathrm{CN})_{3}{ }^{\prime \prime}+\theta \rightleftarrows{ }_{3} \mathrm{CN}^{\prime}+\mathrm{Ag} \text { (metal), }
$$

viz., a direct interchange of negatrons between the $\mathrm{CN}^{\prime}$ ions and the electrode. As before, the reaction from left to right represents the deposition of silver on the cathode.

A specially interesting case is that of the "positive" plate of the lead accumulator. Two hypotheses to explain the reversible reactions on this electrode have found favour, viz., that of Liebenow, which may be shown thus-

$$
\begin{gathered}
\mathrm{PbO}_{2}+2 \theta \rightleftarrows \mathrm{PbO}_{2}^{\prime \prime} \\
\mathrm{PbO}_{2}^{\prime \prime}+4 \mathrm{H} \cdot \stackrel{\mathrm{Pb}}{\longleftrightarrow}+2 \mathrm{H}_{2} \mathrm{O},
\end{gathered}
$$

and that of Le Blanc-

$$
\begin{aligned}
& \mathrm{PbO}_{2}+4 \mathrm{H} \rightleftarrows \mathrm{Pb} \cdots+2 \mathrm{H}_{2} \mathrm{O} \\
& \mathrm{Pb} \cdots+2 \mathrm{P} \rightleftarrows \mathrm{Pb} \cdot .
\end{aligned}
$$

According to Liebenow the formation of $\mathrm{PbO}_{2}^{\prime \prime}$ ions, according to Le Blanc that of $\mathrm{Pb} \cdots$ ions is an essential step in the carrying of the current into or out of the solution. Now the concentration of either of these ions works out to something of the order of $1^{-50}$, so that an application of Haber's reasoning shows that neither of the above hypotheses gives a satisfactory account of what takes place. The reversible reaction on the "positive" electrode is probably simply-

$$
\mathrm{PbO}_{2}+4 \mathrm{H} \cdot+2 \mathrm{O} \rightleftarrows \mathrm{Pb} \cdot+2 \mathrm{H}_{2} \mathrm{O},
$$

viz., a direct interchange of negatrons between the electrode and the hydrions of the electrolyte.

The difference in the mechanism of conduction in elements and compounds must depend on more than the relative closeness of packing of the atoms. In the fused salts, for instance, where the closeness of packing is not greatly less than in metals, conduction must be carried on entirely by ions, seeing that fused salts are transparent and that Faraday's law is obeyed strictly at the electrodes. The negatrons, which pass from the "electropositive" to the "electro-negative" atoms in the process of combination

$$
\text { *Z. Anorg. Ch. 39. } 197 \text { (1904). † †. Elektroch. 10. } 433 \text { (1904). }
$$


between two elements, appear to be too firmly retained by the electronegative atoms to allow of the same kind of dissociation as in metals.

The great need in electrochemistry is a clear idea of the mechanism by which ions are produced in an electrolyte. At present we are almost wholly in the dark here ; and it will be a great triumph for the electron theory if it can bridge this gap.

In conclusion, I think the thanks of the Society are due to Mr. Fournierd'Albe for the highly suggestive Paper which he has brought before it.

Mr. F. Kaye remarked that if it were a fact that the various mobilities of the ions were the result of different hydrations caused by the different condensation powers of the positive and negative ions, it was a result of great interest and importance.

This theory, however, appeared to him to present considerable difficulties for its clear acceptance. The initial cause of the condensation, and therefore of the hydration, was purported to be the electrical charge on the ions. But in a solution the charges in the cations and anions were equal and opposite. What determined the degree of hydration-the nature of the ion or the sign, and amount, of its charge?

The mass of the atoms was evidently not always the determining factor in the mobility of its ion, for in the two groups of atoms $\mathrm{Li}, \mathrm{Na}$, and $\mathrm{K}$ on the one hand, and $\mathrm{Ca}, \mathrm{Sr}$, and $\mathrm{Ba}$ on the other, the heavier ions were the more mobile.

Should not the structure of the atom, or of an ionic group, and therefore the space its hydrated ion occupied, be taken into consideration?

Again, with rising temperatures the mobilities of the ions tend to become the same. Can we say that the power of condensation, due to the electrified nucleus, is weakened at higher temperatures, or that the structural functions are less able to exert themselves?

$\mathrm{He}$ would like to ask Mr. Fournier whether, when a liquid was evaporating, there was a movement of electrons into the vapour, and on the other hand, what happened when the vapour was condensed.

Mr. F. S. Spiers (communicated): In view of the present practical importance of conductors of the solid electrolytic type, it is of interest to note how easily the electron theory explains this form of conduction, and, in particular, the remarkable fact that at high temperatures probably every insulator (unless an element like sulphur, which cannot dissociate) conducts electrolytically. At normal temperatures no free electrons will exist in such bodies, but the effect of raising the temperature will be to cause chemical dissociation of the molecule into two parts, of which one has a greater attraction for the electron than the other. The dissociated parts of the molecule thus become positive and negative ions, and under the influence of an electric field will, like a conducting solution, conduct electrolytically.

It is evident that dissociation is always the necessary preliminary to electrolytic conduction, whether in a solid or liquid. The electron theory, however, does not seem to help explain in what manner this preliminary dissociation takes place when, for example, a salt is dissolved in water.

Dr. J. A. Harker asked the author whether he conceived of atoms as made up entirely of electrons, or whether he regarded them as consisting of a central nucleus with surrounding electrons, something like the solar system. If the former, he thought that there should be a number of intermediate atoms in between the limited number of stable ones known to us, unless it could be proved that certain groupings were more stable than casual grouping would be.

He had heard it from a well-known German physicist that Helmholtz 
once asked Lord Kelvin whether he really believed in the objective truth of the vortex atom theory, and Lord Kelvin answered, "Es war nur ein Traum." Did mathematicians similarly regard the electron theory as merely one out of a number of possible explanations, and did they believe it to be really true? It was really, he thought, most important that the fundamental conceptions of the theory should be perfectly clear and definite, in order that a complete system could be built up, after the fashion of Euclid, on the basis of a few simple axioms and postulates.

The Chairman : I know that I express the unanimous sentiment of this meeting when I say that we feel deeply indebted to Mr. Fournier-d'Albe for the Paper he has communicated to the Society, and for the illuminating oral explanations he has added this evening.

It would be impossible, even if it were desirable, to discharge the obligation his kindness imposes upon us by his coming from Dublin to attend this meeting, but we have made him the best return in our power by subjecting his Paper to trenchant criticism-by doing our best to pull it to pieces; and he has had the satisfaction of seeing how well it has withstood the trying ordeal.

Mr. Fournier-d'Albe could not have chosen a more important or a more interesting subject than the application of the electron theory to explain the phenomena of electrolysis. There is a widespread feeling of the inadequacy of the old ideas to account completely for all the phenomena of electric conduction, electrolytic and other; and yet I confess it is not easy (in the case of a hardened sinner like myself) to get rid of old ideas, and their displacement is a necessary step towards the reception of new-the ideas so vividly expounded by $\mathrm{Mr}$. Fournier-d'Albe. The discussion of this evening is a great help, and when we can ponder the rapidly spoken words more deliberately, as I hope we may be able to do by means of a printed report, much that may on delivery have seemed obscure or wrong will doubtless become clear and free from the seeming error.

Mr. E. E. Fournier d'Albe, in reply, said that he had not been disappointed in his expectations of hearing an interesting discussion.

$\mathrm{He}$ admired Mr. Rhodin for defending Berzelius against Dalton, but he did not think that the few considerations put forward were sufficient to overthrow the electron theory. Mr. Rhodin would, from his particular standpoint, define Jupiter as a conception to explain action at a distance, but the same reasoning and lines of argument used to cxplain the mass of Jupiter were employed in determining the mass and charge of the electron.

In reply to Dr. Borns, the theory was an extension of the ionic theory in the sense that in the latter electricity was regarded as something in the nature of discrete particles, or ions, attached to material particles, in contrast to an etheric theory. The facilitating of condensation by a charge of electricity was in accordance with a well-known principle of energetics.

The Hall effect was explained by supposing that the free paths of the electrons were screwed round in a magnetic field. The reverse swing mentioned by Dr. Borns was not necessarily due to positive electrons, but might be simply caused by positive ions with great mobility. Only a short time previously there was another alleged discovery of positive electrons by Jean Becquerel, but in this case also the existence of positive electrons was not necessarily implied.

With regard to Kaufmann's very delicate expcriments, these merely tested the relative accuracy of the various electron theories : that is to say, they decided the relative claims of the hard and rigid electron (Abraham), the electron which is flying through space becomes an ellipsoid of revolution (Stoney, Lorenz), and the electron which, though flattened, retains its original 
volume (Bucherer), in order to account for the negative results of the Michelson-Morley experiment, and to escape from the dilemma caused thereby. Kaufmann's experiments tended to support the theory of Abraham, but the theory itself certainly did not stand or fall by these experiments. Answering Dr. Harker, not only did the atom, according to Thomson, contain a central positive nucleus, but the latter formed the overwhelming proportion of its total mass, so the analogy to the solar system was a very close one. That there existed not an innumerable number of intermediate atoms was probably due to the fact that only certain configurations were stable, just as in the floating magnet experiments of Professor J. J. Thomson. He could not agree with Dr. Harker's last remarks. The theory was too closely in touch with experimental facts-that was its strength-for the application of rigid methods of deduction from a few simple axioms and postulates.

As regards metallic conduction, he would refer to an article by Professor Schuster in the Philosophical Magazine (February, 1904). There was there evaluated the number of electrons conveying a current through a metal. Referring to the temperature coefficients of the conductivity of electrolytes, it would most likely be found that continuity existed between metals, metalloids, and electrolytes.

He was obliged for the references to work on hydration of ions, and glad that the subject had been so far worked out.

It was self-evident that free electrons could not exist in liquids; their mobility was so great that they would give rise to a conductivity far beyond that actually observed.

Dr. Borns asked what happens to the electrons when you liquefy a conducting solid body. Probably some kind of polymerisation always occurs, due to the greater molecular freedom of the liquid, and therefore ionic groups form. Mercury, for example, has the same order of conductivity in the liquid as in the gaseous state; it was known that the mercury arc owed its conductivity to the free electrons contained in mercury vapour.

With regard to Mr. Wilsmore's suggested term "negatron," it would be inadvisable to stereotype the unfortunate "negative" character of what we know to be the actual electric current in metals. The word " electron" had been coined by Dr. Johnstone Stoney, and had, with rare unanimity, been adopted by practically the whole electrical world.

In reply to Mr. Kaye, the negative ion was the more mobile, probably because it was smaller.

The question of evaporation raised interesting points. A liquid surface, according to osmotic theory, was practically a semi-permeable membrane, and it would behave in a like manner to electrons, which did not evaporate with the vapour. 